${ }^{1}$ L. F. Mollenauer, S. Pan, and S. Yngvesson, Phys. Rev, Lett. 23, 683 (1969).

${ }^{2}$ H. Panepucci and L. F. Mollenauer, Phys. Rev. 178, 589 (1969).

${ }^{3}$ N. V. Karlov, J. Margerie, and Y. Merle-D'Aubigne, J. Phys. (Paris) 24, 717 (1963).

${ }^{4}$ J. Mort, F. Lüty, and F. C. Brown, Phys. Rev. 137, A566 (1965).

${ }^{5} \mathrm{H}$. Mahr, in Physics of Color Centers, edited by
W. B. Fowler (Academic, New York, 1968), p. 270.

${ }^{6}$ H. Seidel, Z. Phys. 165, 218 (1961).

${ }^{7}$ L. F. Mollenauer, S. Pan, and A. Winnacker, to be published.

${ }^{8} \mathrm{H}$. Seidel and H. C. Wolf, in Physics of Color Centers, edited by W. B. Fowler (Academic, New York, $1968)$, p. 555.

${ }^{9}$ W. B. Fowler, in Physics of Color Centers, edited by W. B. Fowler (Academic, New York, 1968), p. 101.

\title{
Influence of Nuclear Forces on Coulomb Fission *
}

\author{
Hartmut Holm and Walter Greiner \\ Institut für Theoretische Physik der Universität, Frankfurt am Main, Germany
}

(Received 1 March 1971)

\begin{abstract}
The Coulomb-fission cross sections for ${ }^{132} \mathrm{Xe}$ and ${ }^{148} \mathrm{Nd}$ incident on ${ }^{238} \mathrm{U}$ are calculated in a dynamical classical model. In particular the influence of nuclear forces on the cross sections is studied. Since they are counteracting the Coulomb force, they diminish the cross sections for Coulomb fission significantly and shift the Coulomb barrier towards lower energies.
\end{abstract}

The time dependence of Coulomb distortions in heavy-ion reactions has been investigated in various articles. $^{1-5}$ In these works the Coulomb barrier has been studied especially carefully. In comparison to it little is known about Coulomb fission. In fact the discrepancies in the theoretical predictions of the Coulomb-fission cross sections are large as can be seen from the work of Wilets, Guth, and Tenn, ${ }^{6}$ and of others. ${ }^{4,7,8}$ In none of these investigations have nuclear forces been considered, though their influence has been realized by the application of the dynamical model on the Coulomb barrier. The short-range nuclear force counteracts the Coulomb force; and, as is seen in Ref. 2, it diminishes the excitation energy of the quadrupole vibrations considerably. One therefore expects that the energy in the fission degree of freedom, and with it the Coulomb-fission cross section, will be lowered when nuclear forces are included in the calculations.

To deal with this effect quantitatively we use the dynamical classical model. As shown by Riesenfeldt and Thomas, ${ }^{4}$ the expectation value of the quadrupole vibrations agrees very well with the classically calculated value of the vibrational amplitude as a function of time. We therefore believe that the classical model is not as inadequate as is claimed by Beyer, Winther, and Smilansky. ${ }^{7}$ Their very small excitation cross sections may be due to the specific assumptions on which their quantum mechanical calculation is based. They do not consider $a_{2}$ vibrations and either neglect rotations ${ }^{7}$ or do not treat them consistently. ${ }^{8}$ It is well known, however, that the Coulomb excitation of rotations is much larger than that of vibrations. Furthermore both degrees of freedom are coupled by the rotation-vibration interaction. We assume that the projectile (1) is spherical, whereas the target nucleus (2) is deformed. Then the total Hamiltonian for central collisions is

$$
H=p_{R}^{2} / 2 m+H_{\mathrm{vib}(1)}+H_{\mathrm{rot}(2)}+H_{\mathrm{vib}(2)}+H_{\mathrm{Cint}}+H_{\mathrm{Yukawa} \text { int }} .
$$

Since the coupling between octupoles and quadrupoles is expected to be small, we restrict ourselves to quadrupole vibrations. Giant resonances are neglected (see Beringer, ${ }^{2}$ Holm et al., ${ }^{9}$ and Eis enberg and Greiner ${ }^{10}$ ). We start with the usual expansion of the nuclear surface in spherical harmonics in the laboratory system,

$$
R(\theta, \varphi)=R_{0}\left[1+\sum_{\mu} \alpha_{2 \mu} Y_{2 \mu}^{*}(\theta, \varphi)\right]
$$

and calculate $H_{\mathrm{C} \text { int }}$ up to terms of second order in the vibrational amplitudes using a constant charge density $\rho_{p}(\overrightarrow{\mathrm{r}})=Z / V$, where $V$ is the nuclear volume. The nuclear radius is given by $R_{0}=r_{0} A^{1 / 3}$ with 
$r_{0}=1.2 \mathrm{fm}$. For the deformed nucleus the $\alpha_{2 \mu}$ are transformed into the intrinsic system:

$$
a_{\nu}=\sum_{\mu} D_{\mu \nu}{ }^{2}(\alpha, \beta, \gamma) \alpha_{2 \mu}, \quad a_{2}=a_{-2}, \quad a_{1}=a_{-1}=0,
$$

where the $\mathbb{D}_{\mu \nu}{ }^{2}$ are the rotational matrices and $\alpha, \beta$, and $\gamma$ the Euler angles. The simplest possible potential for the quadrupole vibrations is the harmonic-oscillator potential. The necessary constants for the projectile are taken from the excitation energy of and the transition probability to the first $2^{+}$ state. For the deformed target nucleus we first use the rotation-vibration model (for details see Refs. 9 and 10). Then $H_{\text {rot(2) }}+H_{\mathrm{vib}(2)}$ is given by

$$
H_{\mathrm{rot}(2)}+H_{\mathrm{vib}(2)}=\sum_{\nu=1}^{3} \frac{L_{\nu}{ }^{2}}{2 J_{\nu}\left(\alpha_{0}, \alpha_{2}\right)}+\frac{1}{2 B}\left(p_{0}{ }^{2}+\frac{1}{2}{p_{2}}^{2}\right)+\frac{1}{2} C_{0}\left(a_{0}-\beta_{0}\right)^{2}+C_{2} a_{2}{ }^{2},
$$

where $p_{0}$ and $p_{2}$ are the canonical conjugate momenta to the amplitudes $a_{0}$ and $a_{2}$. The moments of inertia

$$
J_{1,2}=B\left[2 a_{2}{ }^{2} \pm 2(6)^{1 / 2} a_{2} a_{0}+3 a_{0}{ }^{2}\right], \quad J_{3}=8 B a_{2}{ }^{2}
$$

contain a coupling between the $a_{2}$ and $a_{0}$ vibrations and, if inserted into Eq. (4), lead to the rotation-vibration interaction.

For comparison with the work of Wilets, Guth, and Tenn, ${ }^{6}$ we first solve the classical Hamilton equations with vanishing $H_{\text {Yuka wa int }}$. The $a_{0}$ vibration describes the fission mode with a fission barrier $E_{b}=5.7 \mathrm{MeV}$ for ${ }^{238} \mathrm{U}$. The equilibrium deformation $\beta_{0}$ for ${ }^{238} \mathrm{U}$ is 0.28 . If $a_{0}(t)$ reaches values larger than $\beta_{\mathrm{cr}}=\beta_{0}+\left(2 E_{b} / C_{0}\right)^{1 / 2}$ (see Fig. 1) during the collision process for projectile energies below the Coulomb barrier, we assume that Coulomb fission takes place. It is well known that "soft" vibrations can be excited more easily than "hard" ones. Therefore this simple assumption underestimates the Coulomb-fission cross sections. The barrier and fission cross sections are naturally functions of the initial orientation of the target. The center-of mass

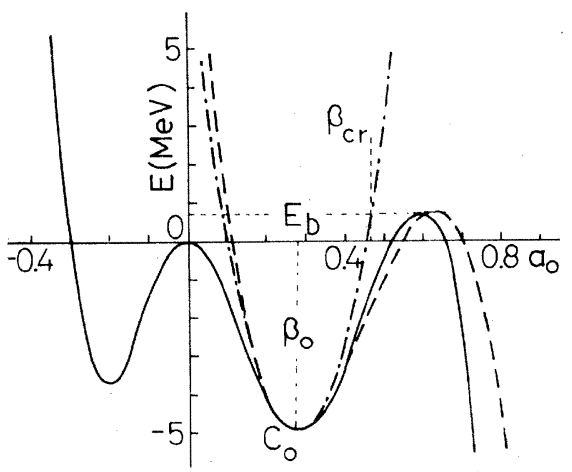

FIG. 1. Comparison between the potential of the $a_{0}$ vibrations in the rotation-vibration model (dash-dotted curve); the cubic potential used by Wilets, Guth, and Tenn (Ref. 6) (dashed curve); and the cut $a_{2}=0$ of the potential-energy surface of ${ }^{238} \mathrm{U} . E_{b}$ describes the height of the fission barrier, $C_{0}$ the stiffness of the $a_{0}$ vibrations, and $\beta_{0}$ the equilibrium deformation. $\beta_{\mathrm{cr}}$ is explained in the text. cross section is

$$
\left(d \sigma_{C f} / d \Omega\right)_{180^{\circ}}=\left(r_{c} / 4\right)^{2} P,
$$

where $\left(r_{c} / 4\right)^{2}$ is the Rutherford cross section and $P$ is the fraction of orientations leading to fission. Analogously, the Coulomb barrier determines the reaction cross section

$$
\left(d \sigma_{R} / d \Omega\right)_{180^{\circ}}=\left(r_{c} / 4\right)^{2} \bar{P},
$$

where $\bar{P}$ is the fraction of orientations leading to an overlap of the surfaces of the projectile and a target nucleus.

The results for ${ }^{132} \mathrm{Xe}$ and ${ }^{148} \mathrm{Nd}$ on ${ }^{238} \mathrm{U}$ are shown in the upper part of Figs. 2 and 3 . It is obvious that for backward scattering, where Coulomb excitation reaches its maximum value, we can restrict ourselves to the Euler angle $\beta$ describing the angle between the nuclear deformation axis and the connection of the two centers of mass. Most favorable for Coulomb fission are initial $\beta$ angles of about $\beta \approx 20^{\circ}$. With increasing energy the cone of favorable $\beta$ angles quickly expands to both sides. Perpendicular orientation of the target, $\beta=\pi / 2$, is the most unfavorable case. Then most of the excitation energy is pumped into the $a_{2}$ mode which in the simple rotation-vibration model is not coupled to the fission mode as the rotations are not excited. The line $\left(d \sigma_{C f} / d \Omega\right)_{180^{\circ}}$ ends when it cuts the line $\left(d \sigma_{R} / d \Omega\right)_{180^{\circ}}$. The Coulomb barrier begins at $0^{\circ}$ and ends with $90^{\circ}$. Then all orientations lead to an overlap of the nuclear surfaces of projectile and target and $\left(d \sigma_{R} / d \Omega\right)_{180^{\circ}}=\left(r_{c} / 4\right)^{2}$. For energies slightly above the beginning of the Coulomb barrier it may be possible to distinguish Coulomb fission from other reaction mechanisms by the fission products. If we compare these results with those of Wilets, Guth, and Tenn ${ }^{6}$, we 


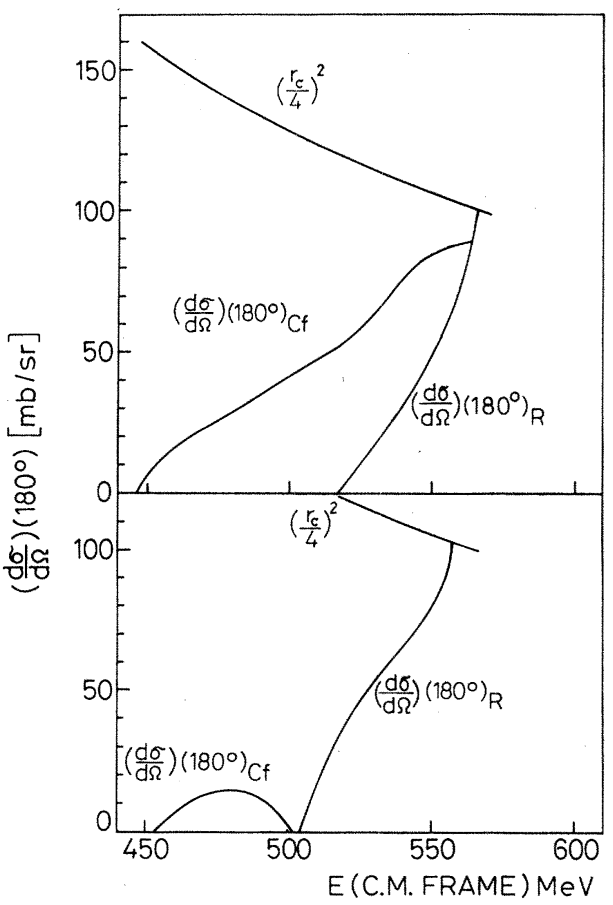

FIG. 2. Differential cross section in the center-ofmass system for Coulomb fission. ${ }^{132} \mathrm{Xe}$ is incident on ${ }^{238} \mathrm{U}$. $\left(r_{c} / 4\right)^{2}$ is the Rutherford cross section for backward scattering. $\left(d \sigma_{R} / d \Omega\right)_{180^{\circ}}$ describes essentially the Coulomb barrier. It is given approximately by the Rutherford cross section multiplied by the probability that the nuclear surfaces of the projectile and a target nucleus come into contact. The upper curves are calculated without a nuclear force. The influence of the Yukawa force (strength constant $V_{0}=-295 \mathrm{MeV} \mathrm{fm}$ ) is shown in the lower curves. The fission cross section is considerably reduced, and the Coulomb barrier is shifted towards lower energies.

first should say that the parameter $\Delta \beta$ in their potential

$$
E\left(a_{0}\right)=3 E_{b}\left(\frac{a_{0}(t)-\beta_{0}}{\Delta \beta}\right)^{2}\left[1-\frac{2}{3}\left(\frac{a_{0}(t)-\beta_{0}}{\Delta \beta}\right)\right]
$$

is not as much undetermined as claimed by the authors. Its value is fixed by the cubic form of the Ansatz. $C_{0}$ is defined as

$$
C_{0}=\left[d^{2} E\left(a_{0}\right) d a_{0}^{2}\right]_{a_{0}=\beta_{0}} \text {. }
$$

With $C_{0}=E_{a_{0}}{ }^{2} / \beta_{0}{ }^{2} E_{\text {rot }}$ we obtain

$$
\Delta \beta=\left(\frac{6 E_{b}}{C_{0}}\right)^{1 / 2}=\frac{\beta_{0}}{E_{a_{0}}}\left(6 E_{b} E_{\mathrm{rot}}\right)^{1 / 2} \approx 0.35,
$$

where $E_{a}$ is the energy of the one-phonon state of the $a_{0}$ vibrations and $E_{\mathrm{rot}}$ is the first rotational level. For $\Delta \beta=0.35$ our results and theirs are of the same order of magnitude.

In the simple rotation-vibration model the fis-

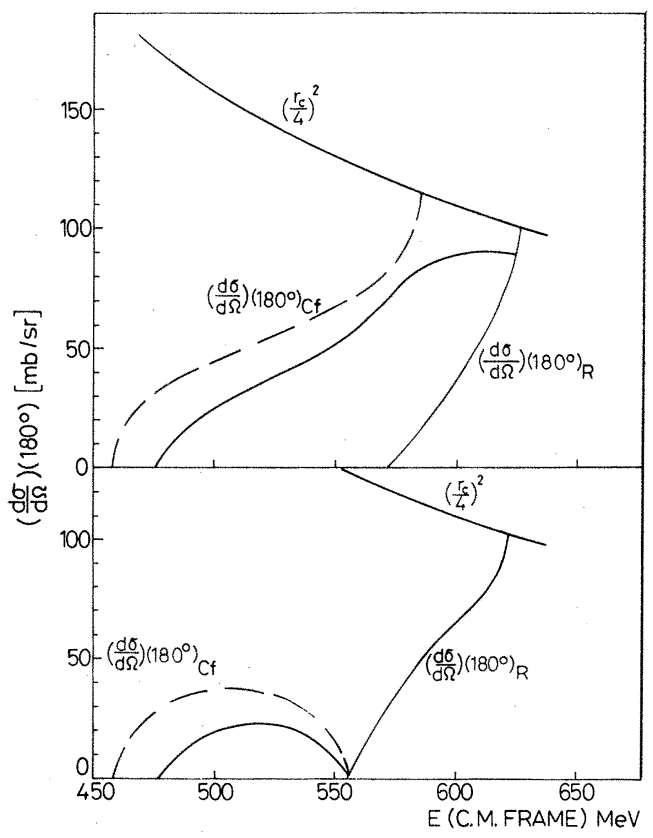

FIG. 3. Coulomb-fission cross section for ${ }^{148} \mathrm{Nd}$ incident on ${ }^{238} \mathrm{U}$. The lower part shows the influence of the Yukawa force (strength constant $V_{0}=-294 \mathrm{MeV} \mathrm{fm}$ ). The cross sections are calculated within the rotationvibration model (solid lines) and with the complete potential energy surface of ${ }^{238} U$ (dashed lines). See caption to Fig. 2.

sion cross section never reaches the curve $\left(r_{c}\right)$ $4)^{2}$ which is especially visible in Fig. 3. Besides, the potential in Eq. (4) does not fulfill the symmetry condition $T\left(\alpha_{0}, \alpha_{2}\right)=\left(\frac{1}{2} a_{0}-\frac{1}{2}(6)^{1 / 2} a_{2}, \frac{1}{2} a_{2}+\frac{1}{2}\right.$ $\left.\times\left(\frac{3}{2}\right)^{1 / 2} a_{0}\right)$ and is therefore restricted to the region $-\left(\frac{3}{2}\right)^{1 / 2} a_{0} \leqslant a_{2} \leqslant\left(\frac{3}{2}\right)^{1 / 2} a_{0}, a_{0} \geqslant 0$, which is not serious since the fission barrier is reached earlier than the limitations (see Fig. 1). This can also be avoided by using a complete potential-energy surface $V\left(a_{0}, a_{2}\right)$ for ${ }^{238} \mathrm{U}$. The rotational invariance of both potentials is discussed in Ref. 10. The $a_{2}=0$ cut, i.e., the potential $V\left(a_{0}, 0\right)$, is the solid line in Fig. 1 . The dashed line is the potential of the $a_{0}$ vibrations used by Wilets, Guth, and Tenn ${ }^{6}$, while the dash-dotted line represents the rotation-vibration model. The potential-energy surface is given by

$$
V\left(a_{0}, a_{2}\right) \sum_{m, n} C_{m n}\left(a_{0}^{2}+2 a_{2}^{2}\right)^{n} a_{0}{ }^{m}\left(6 a_{2}^{2}-a_{0}^{2}\right)^{m}
$$

It has to reproduce correctly the minimum at $\beta_{0}=0.28$, the stiffness parameters $C_{0}$ and $C_{2}$ (curvatures at the minimum in $a_{0}$ and $a_{2}$ direction), and the barrier height $E_{b}=5.7 \mathrm{MeV}$. This can be achieved by using the following coeffi- 
cients:

$$
\begin{aligned}
& C_{01}=-175.8472, \quad C_{10}=-85.72417, \\
& C_{02}=2155.516, \quad C_{11}=3193.989, \\
& C_{03}=-5710.004, \quad C_{20}=1189.203, \\
& C_{12}=-12290.29, \quad C_{04}=274.6812, \\
& C_{21}=-7334.116 .
\end{aligned}
$$

All higher coefficients are zero. The $C_{m n}$ are given in $\mathrm{MeV}$. If the total excitation energy is larger than the maximum of

$$
V_{\text {eff }}\left(a_{0}\right)=V\left(a_{0}, a_{2}=0\right)+\sum_{\nu=1}^{3} \frac{L_{\nu}{ }^{2}}{2 J \nu\left(a_{0}, a_{2}=0\right)},
$$

Coulomb fission takes place. For ${ }^{148} \mathrm{Nd}$ incident on ${ }^{238} \mathrm{U}$ the dashed curve in Fig. 3 shows the result. As expected the Coulomb-fission cross section is raised. The Coulomb barrier is changed very little because near the turning point of the Rutherford hyperbola the excitation energies are small.

Let us now consider the influence of $H_{\text {Yukawa int }}$ in Eq. (1), which is given by

$$
H_{\text {Yukawa int }}=\frac{V_{0}}{4 \pi} \int_{0}^{R_{1}\left(\theta_{1} \varphi_{1}\right)} d \tau_{1} \rho\left(\overrightarrow{\mathrm{r}}_{1}\right) \int_{0}^{R_{2}\left(\theta_{2} \varphi_{2}\right)} d \tau_{2} \rho\left(\overrightarrow{\mathrm{r}}_{2}\right) \frac{\exp \left(-\left|\overrightarrow{\mathrm{r}}_{1}-\overrightarrow{\mathrm{r}}_{2}-\overrightarrow{\mathrm{R}}\right| / \mu\right)}{\left|\overrightarrow{\mathrm{r}}_{1}-\overrightarrow{\mathrm{r}}_{2}-\overrightarrow{\mathrm{R}}\right|}
$$

with $\rho(\overrightarrow{\mathrm{r}})=A / V$ and $\mu=0.8 \mathrm{fm}$. The strength constant $V_{0}$ is evaluated with the same method as described in Ref. 2. The integral (13) is only evaluated up to terms of first order in the deformation parameters. In the calculation of the fission cross section we again use Eq. (6). This is an approximation, because the Rutherford cross section is also changed by the nuclear forces. The results are shown in the lower part of Figs. 2 and 3. At the starting point of $\left(d \sigma_{C f}\right)$ $d \Omega)_{180^{\circ}}$ the influence of nuclear forces is small because the projectile nearly keeps out of their range. At higher energies, however, the Yukawa force counteracts the Coulomb force more and more. Therefore the fission cross section no longer increases with increasing energy in this region. Thus near the barrier the Coulomb-fission cross sections are much smaller than those calculated without a nuclear force. Also $\left(d \sigma_{R}\right)$ $d \Omega)_{180^{\circ}}$ changes its shape somewhat and is shifted towards lower energies.

Both effects, the deformation of the target and the Yukawa force, are usually simulated by calculating the Coulomb barrier for rigid spheres using a larger radius constant. But they are not sufficient to explain completely the experimental values of $r_{0}=1.4-1.45 \mathrm{fm}$. In fact, the experimental values for $r_{0}$ can be reproduced by using $r_{0}=1.35 \mathrm{fm}$ in our model. Then in the ${ }^{132} \mathrm{Xe}^{238} \mathrm{U}$ case the cross sections are shifted about $50 \mathrm{MeV}$ towards lower energies. If higher-multipole vibrational modes are included into the calculations, this discrepancy probably disappears. Near the barrier it is possible that the nuclear forces counteract the Coulomb force so strongly that the excitation energy of the vibrations is higher than the fission barrier after the collision process (Yukawa fission). For ${ }^{40} \mathrm{Ar}$ and ${ }^{84} \mathrm{Kr}$ there is no Coulomb fission.

In conclusion we want to thank Dr. W. Scheid, Professor G. T. Emery, Professor R. G. Newton, and Professor G. E. Walker for valuable discussions. We also gratefully acknowledge the opportunity to work at the physics department of Indiana University, Bloomington, and the kind hospitality extended to us there.

\footnotetext{
*Work supported by the Bundesministerium für Bildung und Wissenschaft and the Deutsche Forschungsgemeinschaft.

${ }^{1}$ R. Beringer, Phys. Rev. Lett. 18, 1006 (1967).

${ }^{2}$ H. Holm and W. Greiner, Phys. Rev. Lett. 24, 404 (1970).

${ }^{3}$ A. S. Jensen and C. Y. Wong, Phys. Rev. C 1 , 1321 (1970).

${ }^{4}$ P. W. Riesenfeldt and T. D. Thomas, Phys. Rev. C 2, 711 (1970).

${ }^{5}$ A. S. Jensen and C. Y. Wong, Phys. Lett. 32B, 567 (1970).

${ }^{6}$ L. Wilets, E. Guth, and J. S. Tenn, Phys. Rev. 156, 1349 (1967).

${ }^{7} \mathrm{~K}$. Beyer and A. Winther, Phys. Lett. 30B, 296 (1969).

${ }^{8} \mathrm{~K}$. Beyer, A. Winther, and U. Smilanski, in Proceedings of the Intermational Conference on Nuclear Reactions Induced by Heavy Ions, Heidelberg, Germany, 1969, edited by R. Bock and R. Herring (NorthHolland, Amsterdam, 1970).

${ }^{9}$ H. Holm, D. Scharnweber, W. Scheid, and W. Greiner, Z. Phys. 231, 450 (1970).

${ }^{10} \mathrm{~J}$. Eisenberg and W. Greiner, Nuclear Theory (North-Holland, Amsterdam, 1970), Vol. I.
} 